\title{
Sarcoma by FNCLCC Grade
}

National Cancer Institute

\section{Source}

National Cancer Institute. Sarcoma by FNCLCC Grade. NCI Thesaurus. Code C9023.

Grading of sarcomas according to the French Federation of Cancer Centers Sarcoma Group (FNCLCC). This sarcoma grading system is based on three factors: differentiation, mitotic count, and tumor necrosis. The scores for each factor are added to determine the grade (1 to 3 ) for the sarcoma. 